\title{
In Vivo Evaluation of Jatropha curcas L (Euphorbiaceae) Leaves Acute and Subacute Toxicity in Mice
}

\author{
S. Sawadogo ${ }^{1}$, S. D. Sanou ${ }^{1 *}$, A. P. Dabiré ${ }^{1}$, G. R. Belemtougri ${ }^{1}$, L. Sawadogo ${ }^{1}$, J. de Leiris ${ }^{2}$, S. \\ Tanguy $^{2}$, F. Boucher ${ }^{2}$ \\ ${ }^{1}$ Laboratory of Animal Physiology, University Ouaga I Pr Joseph KI-ZERBO, Burkina Faso, 03 BP \\ 7021 Ouaga 03 \\ ${ }^{2}$ Equipe cœur et nutrition, TIMC-PRETA UMR 5525, Université de Grenoble, France
}

Received 27 February 2018, accepted in final revised form 15 April 2018

\begin{abstract}
Jatropha curcas is a medicinal plant whose dead leaves are particularly used in arterial hypertension and diabetes treatment. The purpose of this study is to conduct an acute and subacute toxicity study of Jatropha curcas leaves aqueous extract (JCLAE) using OECD method. For acute toxicity, dose limits of 2000 and $5000 \mathrm{mg} / \mathrm{kg}$ were used. In subacute toxicity study 4 batches were constituted including a control batch that received distilled water for 28 days and the other 3 batches, JCLAE doses of respectively 200, 400, 800 $\mathrm{mg} / \mathrm{kg}$ for the same duration. The $\mathrm{LD}_{50}$ was determined and the hematological, biochemical and histological parameters were analyzed in mice. The $\mathrm{LD}_{50}$ is greater than $5000 \mathrm{mg} / \mathrm{kg}$. HDL-C is the only biochemical parameter that has experienced significant rise variation. Hematological analysis showed a decrease in mean platelet volume and Platelets number. The histological study revealed cases of hepatic cellular apoptosis and kidneys tubular necrosis among animals treated with highest dose. JCLAE is less toxic than Jatropha curcas leaves aqueous extract and high-dose JCLAE also has a moderate toxic effect on thrombocyte line and a protective effect on cardiovascular system.
\end{abstract}

Keywords: Jatropha curcas; Cardiovascular system; Toxicity; Apoptosis.

(C) 2018 JSR Publications. ISSN: 2070-0237 (Print); 2070-0245 (Online). All rights reserved. doi: http://dx.doi.org/10.3329/jsr.v10i2.35267 J. Sci. Res. 10 (2), 187-193 (2018)

\section{Introduction}

Traditional medicine offers recipes based on medicinal plants and some of them may be toxic. Jatropha curcas is a medicinal plant whose leaves are used in many pathologies treatment. In Cameroon Jatropha curcas leaves are used to treat arthritis and abscess in stomach [1]. In Benin this leaf decoction is used to treat edema and cough [2].

*orresponding author: dsanou@univ-ouaga.bf; sanoudrissa82@yahoo.fr 
In Burkina Faso Jatropha curcas is used to treat several diseases such as sickle cell disease, diabetes, gout, tumors, oral candidiasis, skin diseases, malaria, intestinal parasitosis, fever cases, and liver disorders. The dead leaves are particularly used to treat arterial hypertension and diabetes [3].

Jatropha curcas is a shrub that can be reproduced by seeds and cuttings. It is believed to be native to tropical America and is often found in wetlands, in tropical countries stream banks. Despite this plant various virtues and its use in traditional medicine, very few studies were focused on its toxicity. To contribute to the plant innocuousness establishment, we have carried out this acute and subacute toxicity study of its leaves aqueous extract.

\section{Materials and Method}

\subsection{Plant material}

Jatropha curcas leaves yellowing on the plant were collected at Kombissiri, a village situated at $45 \mathrm{~km}$ from Ouagadougou (Burkina Faso). This identification was carried out by the Plant Biology and Physiology Laboratory of the University of Ouaga I Pr. Joseph KI-ZERBO. Those Jatropha curcas leaves were dried in shade for fourteen days and then ground using an electric grinder to obtain a powder which was sieved.

\subsection{Leaves total aqueous extract preparation}

$150 \mathrm{~g}$ vegetable powder was added and homogenized in $1500 \mathrm{~mL}$ of distilled water, and left to macerate with magnetic stirring for $24 \mathrm{~h}$ at room temperature.

Then the macerate was filtered three times on hydrophilic cotton, then lyophilized and shielded from light in non-transparent flasks. The yield was $8.2 \%$.

Yield $=($ Mass of extract $/$ mass of dry plant $) \times 100$

\subsection{Animal material}

9 week-old NMRI strain mice, weighing between 25 and $35 \mathrm{~g}$ were used to test acute and subacute toxicity. These male and female mice were taken from UFR/SVT breeding farm at the University of Ouaga I Pr Joseph KI-ZERBO. The mice were raised in stable temperature rooms $\left(24 \pm 2{ }^{\circ} \mathrm{C}\right)$ where they had free access to water and granular.

\subsection{Acute toxicity}

An orientation study was initially carried out and consisted in dividing 6 female mice into 2 batches of 3 . The first and second batches respectively received a unique dose of 2000 $\mathrm{mg} / \mathrm{kg}$ and $5000 \mathrm{mg} / \mathrm{kg}$ by tube-feeding using a probe. A main study was conducted following that orientation study with a test limit dose of $5000 \mathrm{mg} / \mathrm{kg}$ in accordance with 
OECD Guideline 420 [4]. These mice were fasted $4 \mathrm{~h}$ before extract administration and 1 $\mathrm{h}$ after they were observed for 2 weeks to record any behavioral changes and to count the dead to determine lethal dose $50\left(\mathrm{LD}_{50}\right)$.

\subsection{Subacute toxicity}

The subacute toxicity study was conducted in accordance with the OECD protocol 407 [5,6]. Mice were divided into 4 batches of 8 animals each ( 4 males and 4 females). Three batches were injected daily through tube-feeding for 28 days with Jatorpha curcas leaves aqueous extracts, JCLAE (200, 400 and $800 \mathrm{mg} / \mathrm{kg}$ ). A control batch was treated with distilled water over the same period. Each animal's weight was taken at the beginning and every week until the treatment end.

After 28 days each animal was anesthetized with ketamine plus xylazine as 2 volumes of ketamine for 1 volume of xylazine and administered at a dose of $1 \mathrm{ml} / \mathrm{kg}$. Blood sample was taken in a tube containing an anticoagulant (K3EDTA) to determine hematological and biochemical parameters $[7,8]$. These haematological parameters were evaluated by a mindray BC-3000Plus brand haematology counter: White Blood Cells or Leukocytes (WBC), lymphocytes (lymph), les Granulocytes (Gran), les Monocytes (mono), Red Blood Cell (RBC), Hemoglobin (HGB), Hematocrit (Ht), Mean Corpuscular Volume (MCV), Mean Corpuscular Hemoglobin (MCH), Mean Corpuscular Hemoglobin concentration ( MCHC), Platetet (PLT) and Mean platelet volume (MPV).

These biochemical parameters were determined using RMS/BCA 201 spectrophotometer: Creatinine, Alanine aminotransferase (ALAT), Aspartate aminotransferase (AST), according to kinetic method; urea, total proteins (TP), total Cholesterol ( $\mathrm{T}$ cholest), Triglycerides (TG), High Density Lipoprotein (HDL), by colorimetric method. Low Density Lipoproteins (LDL) was determined by Friedewald formula. Glucose was measured using the Codefree Glucose Meter. The kidneys, liver, heart, lungs and spleen were removed and weighed. The kidneys and liver were fixed with $10 \%$ formalin for histopathological examinations.

\subsection{Statistical analysis}

The results of statistical analyzes were carried out using Graph Pad Prism 5.00 software. Values are presented as mean \pm standard error on average. Significance degrees between treated and control batches were measured by one way analysis of variance (ANOVA) followed by Dunnett's $t$-test to compare mean effect among different groups to that of control groups. If $\mathrm{p}<0.05$, the difference is considered significant. 


\section{Results}

\subsection{Acute toxicity}

Acute toxicity results did not show any sign of toxicity following administration of JCLAE by oral route dose $5000 \mathrm{mg} / \mathrm{kg}$ body weight (bw). All mice survived after 14 days of testing. JCLAE $\mathrm{LD}_{50}$ is therefore greater than $5000 \mathrm{mg} / \mathrm{kg}$ of body weight.

\subsection{Subacute toxicity}

No abnormal behavior was observed over 28 days of treatment. Their body weight did not change significantly with time (Table 1), as well as the relative weights of organs taken at the end of treatment (Table 2).

The haemogram did not show a significant change in the number of white and red blood cells. In contrast, mean platelet volume decreased significantly at $800 \mathrm{mg} / \mathrm{kg} \mathrm{BW}$ dose (Table 3). Platelets number has decreased significantly for doses $400 \mathrm{mg} / \mathrm{kg}$ and 800 $\mathrm{mg} / \mathrm{kg}$ compared with control batch. The results presented in Table 4 show that the JCAE has been without significant effect on certain plasma parameters such as: glucose, creatinine, urea, transaminases as well as total proteins, total cholesterol and LDL, triglycerides. However, HDL-C increased very significantly (Table 4).

Liver histopathological analyses show regular hepatocytes, numerous apoptotic patterns and extensive lesions of punctate lobular necrosis with vascular congestion and hemorrhagic suffusion consistent with moderate hepatotoxicity. Kidney histopathological examination shows lesions of subacute interstitial nephritis with vascular congestion, consistent with toxic etiology.

Table 1. Jatropha curcas leaves aqueous extract effect on mice body weight over 28 days of treatment.

\begin{tabular}{cccccc}
\hline $\begin{array}{c}\text { Doses }(\mathrm{mg} / \mathrm{kg} \\
\text { de PC) }\end{array}$ & 0 & 7 & 14 & 21 & 28 \\
\hline 0 & $33,5 \pm 1,66$ & $33,62 \pm 1,64$ & $33,87 \pm 1,90$ & $34,25 \pm 1,82$ & $33,6 \pm 1,82$ \\
200 & $32 \pm 1,53$ & $32,37 \pm 1,75$ & $33,37 \pm 1,88$ & $33 \pm 1,93$ & $32,82 \pm 1,98$ \\
400 & $31 \pm 1,26$ & $30,87 \pm 1,56$ & $31,62 \pm 1,63$ & $31,75 \pm 1,52$ & $30,81 \pm 1,41$ \\
800 & $31,25 \pm 1,55$ & $29,12 \pm 2,13$ & $30,12 \pm 1,85$ & $30,37 \pm 1,63$ & $30,3 \pm 1,65$ \\
\hline
\end{tabular}

Table 2. Jatropha curcas aqueous extract effects on relative weight of organs taken from mice after 28 days of treatment.

\begin{tabular}{ccccccccc}
\hline Doses (JCLAE) & \multicolumn{2}{c}{$0 \mathrm{mg} / \mathrm{kg}$} & \multicolumn{2}{c}{$200 \mathrm{mg} / \mathrm{kg}$} & \multicolumn{2}{c}{$400 \mathrm{mg} / \mathrm{kg}$} & \multicolumn{2}{c}{$800 \mathrm{mg} / \mathrm{kg}$} \\
\hline Liver & $3,79 \pm$ & 0,06 & $3,85 \pm$ & 0,15 & $3,84 \pm 0,09$ & $4,31 \pm$ & 0,44 \\
Heart & $0,45 \pm$ & 0,03 & $0,38 \pm$ & 0,02 & $0,44 \pm$ & 0,02 & $0,45 \pm$ & 0,01 \\
Lungs & $0,54 \pm$ & 0,02 & $0,55 \pm$ & 0,03 & $0,66 \pm$ & 0,04 & $0,79 \pm$ & 0,08 \\
Spleen & $0,48 \pm$ & 0,08 & $0,45 \pm$ & 0,06 & $0,43 \pm$ & 0,04 & $0,46 \pm$ & 0,06 \\
Kidneys & $1,15 \pm$ & 0,06 & $1,08 \pm$ & 0,09 & $1,15 \pm$ & 0,05 & $1,10 \pm$ & 0,03 \\
\hline
\end{tabular}


Table 3. Jatropha curcas aqueous extract effects on blood figurative elements taken from mice after 28 days of treatment.

\begin{tabular}{ccccc}
\hline Doses (JCLAE) & $0 \mathrm{mg} / \mathrm{kg}$ & $200 \mathrm{mg} / \mathrm{kg}$ & $400 \mathrm{mg} / \mathrm{kg}$ & $800 \mathrm{mg} / \mathrm{kg}$ \\
\hline WBC (10^9/L) & $4,4 \pm 0,28$ & $4,74 \pm 1,14$ & $3,84 \pm 0,41$ & $3,46 \pm 0,56$ \\
Lymph (\%) & $71,4 \pm 1,89$ & $71,2 \pm 2,42$ & $70,2 \pm 1,56$ & $67,4 \pm 2,69$ \\
Gran (\%) & $25,4 \pm 1.80$ & $25,6 \pm 2,38$ & $26,8 \pm 1,46$ & $30 \pm 2,34$ \\
Mono (\%) & $3,2 \pm 0,58$ & $3,2 \pm 0,37$ & $3 \pm 0,32$ & $2,8 \pm 0,37$ \\
RBC (10^12/L) & $7,09 \pm 0,32$ & $7,48 \pm 0,31$ & $7,67 \pm 0,22$ & $7,24 \pm 0,12$ \\
HGB (g/dl) & $11,32 \pm 0,53$ & $11,64 \pm 0,59$ & $12,5 \pm 0,32$ & $11,4 \pm 0,32$ \\
HCT (\%) & $34,22 \pm 1,17$ & $35,18 \pm 1,19$ & $36,08 \pm 1,00$ & $33,76 \pm 0,78$ \\
MCV (Fl) & $48,4 \pm 0,65$ & $47,14 \pm 0,56$ & $47,26 \pm 0,31$ & $46,6 \pm 0,39$ \\
MCH (pg) & $15,9 \pm 0,17$ & $16,1 \pm 0,69$ & $16,26 \pm 0,07$ & $15,66 \pm 0,19$ \\
MCHC (g/Dl) & $33 \pm 0,60$ & $32,96 \pm 0,70$ & $34,48 \pm 0,24$ & $33,72 \pm 0,36$ \\
PLT (10^9/L) & $770 \pm 74,99$ & $671,4 \pm 72,37$ & $527 \pm 76,17 *$ & $532,2 \pm 31,18 *$ \\
MPV (fL) & $7,04 \pm 0,22$ & $6,06 \pm 0,32$ & $6,52 \pm 0,42$ & $5,74 \pm 0,17 *$ \\
\hline
\end{tabular}

White Blood Cells or Leukocytes(WBC), lymphocytes (lymph), les Granulocytes (Gran), les Monocytes (mono), Red Blood Cell (RBC), Hemoglobin (HGB), Hematocrit (Ht), Mean Corpuscular Volume (MCV), Mean Corpuscular Hemoglobin (MCH), Mean Corpuscular Hemoglobin concentration ( MCHC), Platetet (PLT) and Mean platelet (MPV).

Table 4. Jatropha curcas aqueous extract effects on mice biochemical parameters after 28 days of treatment.

\begin{tabular}{lllll}
\hline Doses $(\mathrm{EAFJC})$ & $0 \mathrm{mg} / \mathrm{kg}$ & $200 \mathrm{mg} / \mathrm{kg}$ & $400 \mathrm{mg} / \mathrm{kg}$ & $800 \mathrm{mg} / \mathrm{kg}$ \\
\hline Glucosa $(\mathrm{mmol} / \mathrm{L})$ & $13,12 \pm 1,33$ & $13,63 \pm 1,53$ & $14,88 \pm 0,95$ & $15,56 \pm 0,92$ \\
Creatinine $(\mu \mathrm{mol} / \mathrm{L})$ & $43,1 \pm 9,44$ & $35,66 \pm 4,43$ & $42,44 \pm 5,17$ & $33,3 \pm 3,71$ \\
Urea $(\mathrm{mmol} / \mathrm{L})$ & $8,56 \pm 0,97$ & $7,98 \pm 0,53$ & $7,77 \pm 0,55$ & $8,56 \pm 1,01$ \\
ALAT $(\mathrm{UI} / \mathrm{L})$ & $147,54 \pm 21,21$ & $122 \pm 26,26$ & $148,52 \pm 27,17$ & $160,16 \pm 29,50$ \\
ASAT $(\mathrm{UI} / \mathrm{L})$ & $233,26 \pm 22,29$ & $182,22 \pm 25,60$ & $233,6 \pm 11,27$ & $189,04 \pm 26,60$ \\
$\mathrm{P}$ T $(\mathrm{mg} / \mathrm{dL})$ & $5,25 \pm 0,21$ & $5,08 \pm 0,07$ & $5,42 \pm 0,28$ & $5,28 \pm 0,17$ \\
Cholest T $(\mathrm{mmol} / \mathrm{L})$ & $1,71 \pm 0,18$ & $1,65 \pm 0,25$ & $1,87 \pm 0,24$ & $2,45 \pm 0,39$ \\
T G $(\mathrm{mmol} / \mathrm{L})$ & $0,72 \pm 0,10$ & $0,75 \pm 0,16$ & $0,93 \pm 0,15$ & $0,51 \pm 0,08$ \\
HDL $(\mathrm{mmol} / \mathrm{L})$ & $0,19 \pm 0,02$ & $0,17 \pm 0,07$ & $0,27 \pm 0,05$ & $0,49 \pm 0,05 * *$ \\
LDL $(\mathrm{mmol} / \mathrm{L})$ & $1,19 \pm 0,18$ & $1,13 \pm 0,16$ & $1,17 \pm 0,22$ & $1,73 \pm 0,33$ \\
\hline
\end{tabular}

Glucosa, Creatinine, urea, Alanine aminotransferase (ALAT), Aspartate aminotransferase (AST), total proteins (TP), total Cholesterol (T cholest), Triglycerides (TG), High Density Lipoprotein (HDL), Low Density Lipoproteins (LDL)

\section{Discussion}

Oral acute toxicity study of JCLAE showed a lethal dose $50\left(\mathrm{LD}_{50}\right)$ greater than 5000 $\mathrm{mg} / \mathrm{kg}$ body weight. Dabiré et al. [9]; Nga et al. [10] and Ping et al. [11] used the same method and showed that $\mathrm{LD}_{50}$ of Excoecaria grahamii (Euphorbiaceae) and Alchornea cordifolia (Euphorbiaceae) leaves aqueous extract; and Euphorbia hirta L (Euphorbiaceae) methanolic extract are greater than $5000 \mathrm{mg} / \mathrm{kg}$ body weight.

Studies conducted by Dangambo et al. [12] on Jatropha curcas leaves aqueous extract acute toxicity showed an $\mathrm{LD}_{50}$ of $2792.85 \mathrm{mg} / \mathrm{kg}$ body weight. This result shows that dead leaves aqueous extract would be less toxic than Jatropha curcas leaves aqueous extract. 
Hematological analyses showed that total Jatropha curcas leaves aqueous extract did not cause significant changes in the number of erythrocytes and leucocytes. On the other hand, platelets number and mean platelet volume decreased significantly between batch I, batch III and IV respectively, and between batch I and batch IV. The decrease in MPV and PLT indicates that JCLAE has a toxic effect on platelet line. Mukinda and Eagles [13] reported that Polygala fruticosa extract causes a decline in platelet production.

Biochemical analyses showed a very significant increase in HDL-C of treated batch $(800 \mathrm{mg} / \mathrm{kg}$ ) compared with the control ones.

Angiotensin converting enzyme (ACE) inhibitors are known to increase in HDL-C [14]. Segura-Campos et al. [15] have shown that a peptide isolated from Jatropha curcas has an angiotensin converting enzyme inhibitory activity. HDL-C increase in our study could be related to this molecule presence in JCLAE.

HDL-C, also known as "good cholesterol", is a High Density Lipoprotein (HDL) that transports cholesterol from the tissues to the liver for elimination, therefore preventing accumulation of cholesterol on artery walls, thus decreasing atherosclerosis risk [16]. JCLAE may contain protective substances for cardiovascular system.

Apoptosis or programmed cell death is an important process in regulating cellular homeostasis and may limit tumor growth [17]. Jatropha curcas leaves anticancer properties have been demonstrated by Balaji et al. [18]. Molecules with anticancer properties were isolated from Jatropha curcas leaves [19,20]. These molecules anticancerous activity could justify the high amount of apoptotic features in the liver histopathological analysis. Jatropha curcas leaves still retain their anticancerous properties. Subacute interstitial nephritis lesions with vascular congestion observed in the kidney histopathological analysis show that JCLAE at $800 \mathrm{mg} / \mathrm{kg}$ has a toxic effect on kidneys.

\section{Conclusion}

JCLAE acute and subacute toxicity study by oral route showed that this extract is less toxic than green leaves aqueous extract and this justifies its use in traditional medicine. In addition, this extract may have a protective effect on cardiovascular system and retain its anticancerous properties. However, at high dose JCLAE has a toxic effect on thrombocyte number, liver and kidneys. Further work is needed to confirm its angiotensin converting enzyme inhibitory activity.

\section{References}

1. J. M. Watt and M. G. Breyer-Brandwijk, $2^{\text {nd }}$ Edition (S. Livingstone, Ltd., Edindurgh, 1962) pp. 420-422.

2. H. D. Neuwinger (Chapman and Hall, New York, 1996) pp. 500-509.

3. O. G. Nacoulma / Ouedraogo, PhD thesis, Univ. Ouagadougou, France (1996).

4. Organization of Economic Co-Operation and Development (OECD), OECD Guideline for Testing of Chemicals: 420-Acute Oral Toxicity, OECD, Paris (1001) pp. 1-14. 
5. OCDE. Étude de toxicité orale à dose répétée pendant 28 jours sur les rongeurs. In Lignes directrice de l'OCDE pour les essais de produits chimiques. OCDE, (2008) pp 1-14.

6. B. Gandhare, S. Kavimani, and B. Rajkapoor. J. Sci. Res. 5, 315 (2013) http://dx.doi.org/10.3329/jsr.v5i2.11800

7. E. Sacchetto, D. Ali, E. Dumontet, D. Le carrer, J. - L. Orsonneau, O. Delaroche, and E. BigotCorbel, Ann. Biol. Clin. 72, 337 (2014).

8. H. Kamali, M. Mohri, Revue Méd. Vét. 166, 275 (2015).

9. A. P. Dabiré, Y. Ouedraogo, R. G. Belemtougri, S. Sawadogo, and M. Tiendrebeogo, Int. J. Pharm. Sci. Res. 2, 12 (2017).

10. E. N. Nga, J. Yinyang, E. Baran, G. Etame-Loe, and S. D. Dibong, J. Appl. Biosci. 109, 10649 (2017). http://dx.doi.org/10.4314/jab.v109i1.9

11. K. Y. Ping, I. Darah, Y. Y. Chen, S. Sreeramanan, and S. Sasidharan, BioMed. Res. Int. Article ID 18206 (2013) https://doi.org/10.3390/molecules17077782

12. M. A. Dangambo, H. T. Kabara, A. J. Alhassan, M. S. Sule, M. K. Atiku, A. M. Wudil, L. A. Mashi, Brit. J. Pharm. Res. 5, 52 (2015). https://doi.org/10.9734/BJPR/2015/13105

13. J. T. Mukinda and P. F. Eagles, J. Ethnopharmacol. 128, 236 (2010). https://doi.org/10.1016/j.jep.2010.01.022

14. D. Gude, J. Sci. Soc. 41, 59 (2014).

15. M. R. Segura-Campos, F. Peralta-Gonzalez, A. Castellanos-Ruelas, L. A. Chel-Guerrero, and D. A. Betancur-Ancona, BioMed. Res. Int. Article ID 541947 (2013). http://dx.doi.org/10.1155/2013/541947

16. M. Arock, K. Chevet, R. Couderc, A. Del Corso, V. Ducros, R. Garnotel, I. Gastin, P. Gillery, N. Kapel, L. Kramer, A. Legrand, G. Le moël, C. Morin, C. Queyrel, J. C. Renversez, N. Schneider, P. Therond, H. Tronel, I. Villena, and J. P. Zarski, Le guide des examens biologiques, Le Quotidien du Pharmacien (2008).

17. C. M. Payne, H. Bernstein, C. Bernstein, and H. Grewal, Ultrastruct. Pathol. 19, 221 (1995). https://doi.org/10.3109/01913129509064227

18. R. Balaji, N. Rekha, M. Deecaraman, and L. Manikandan, Afric. J. Pharmacy Pharmacol. 3, 547 (2009).

19. R. Staubmann, M. Schubert-Zsilavecz, A. Hiermann, and T. Kartnig, Phytochem. 50, 337 (1999). https://doi.org/10.1016/S0031-9422(98)00531-7

20. S. A. Al-ssa, Saudi Pharm. J. 21, 305 (2013). https://doi.org/10.1016/j.jsps.2012.09.002 